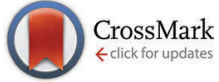

Cite this: Chem. Commun., 2016, 52,190

Received 27th August 2015, Accepted 22nd October 2015

DOI: $10.1039 / c 5 c c 07197 f$

www.rsc.org/chemcomm

\section{Spontaneous reaction between an uncharged lithium iron silicate cathode and a $\mathrm{LiPF}_{6}$-based electrolyte}

\author{
Zachary Arthur, ${ }^{a}$ Hsien-Chieh Chiu, ${ }^{\mathrm{b}}$ Xia Lu, ${ }^{\mathrm{b}}$ Ning Chen, ${ }^{\mathrm{C}}$ Vincent Emond, ${ }^{\mathrm{a}}$ \\ Karim Zaghib, ${ }^{d}$ De-Tong Jiang ${ }^{* a}$ and George P. Demopoulos*b
}

The reaction between an uncharged $\mathrm{Li}_{2} \mathrm{FeSiO}_{4}$ (LFS) cathode and a $\mathrm{LiPF}_{6}-\mathrm{EC} / \mathrm{DMC}$ electrolyte is revealed by in situ XANES in coin cells. This study shows clear evidence of delithiation and iron oxidation in LFS prior to cycling. Subsequent cycling appears to partially restore the original lithiation level, an observation that needs to be taken into consideration in future LFS development work.

Today, lithium-ion batteries (LIBs) are ubiquitous; they can be found everywhere from our portable electronics, to the automotive and renewable energy sector. ${ }^{1,2}$ Indeed, LIBs have been a crucial enabling component in the development of many of the transformative technologies we have seen emerging since the early $2000 \mathrm{~s}$, e.g. smart phones, electric cars, etc. In order to improve the current generation of LIBs it is necessary to develop low cost, safe, and high energy density cathode materials. One material class that has been garnering great interest in this respect is that of $\mathrm{Li}_{2} \mathrm{MSiO}_{4}$ (LMS, $\mathrm{M}=\mathrm{Fe}, \mathrm{Mn}$ ) silicates, an olivine structure that has a theoretical charge capacity of $330 \mathrm{~mA} \mathrm{~h} \mathrm{~g}{ }^{-1,3,4}$ double of that of lithium iron phosphate ${ }^{5}$.

The challenges currently facing the development of LFS $(\mathrm{M}=\mathrm{Fe})$ cathodes are not trivial. The charge compensation mechanism for $\mathrm{Li}_{1-x} \mathrm{FeSiO}_{4}, 0<x<1$, is still not fully understood, ${ }^{6,7}$ and the extraction of more than one lithium results in capacity loss, attributed to $\mathrm{Fe}-\mathrm{Li}$ anti-site defects and phase transitions induced during charging-discharging. ${ }^{6,8}$ Meanwhile, the compatibility between the electrolyte and the cathode may be a cause of concern as well, ${ }^{9,10}$ especially in this case because the fluorine in the typical, most popular $\mathrm{LiPF}_{6}$-based electrolyte could react with the Si species in LFS. ${ }^{11-13}$ However, the preceding studies have either looked into the LFS-electrolyte interaction issue by post mortem (i.e. after cycling) ex situ surface characterization using techniques like $\mathrm{XPS}^{12}$ or by bulk characterization using

\footnotetext{
${ }^{a}$ Department of Physics, University of Guelph, 50 Stone Road East, Guelph, ON N1G 2W1, Canada. E-mail: djiang@uoguelph.ca

${ }^{b}$ Materials Engineering, McGill University, Montreal, QC H3A oC5, Canada. E-mail: george.demopoulos@mcgill.ca

${ }^{c}$ Canadian Light Source, 44 Innovation Boulevard, Saskatoon, SK S7N 2V3, Canada

${ }^{d}$ Institut de Recherche d'Hydro-Québec (IREQ), Varennes, QC J3X 1S1, Canada
}

XRD and EDX following aggressive ageing at $60{ }^{\circ} \mathrm{C} .{ }^{11}$ Understanding the electrode-electrolyte interphasial phenomena as proven in the case of the well-known solid-electrolyte interphase (SEI) $)^{9,14}$ is crucial for the development of robust silicate cathode Li-ion batteries. In particular in situ probing of these phenomena can provide critical missing links in this regard.

In this study, the spontaneous reaction between an uncharged (prior to cycling) $\mathrm{Li}_{2} \mathrm{FeSiO}_{4}$ (LFS) cathode and a $\mathrm{LiPF}_{6}-\mathrm{EC} / \mathrm{DMC}$ electrolyte is studied by in situ X-ray absorption near-edge spectroscopy (XANES) using coin cells. The LFS cathode (80\% LFS, $10 \%$ acetylene black and $10 \%$ PVDF) featured $\mathrm{Li}_{2} \mathrm{FeSiO}_{4}$ nanoparticles prepared via an organic-assisted hydrothermalannealing ( $180{ }^{\circ} \mathrm{C}$ and $400{ }^{\circ} \mathrm{C}$, respectively) synthesis method. ${ }^{15}$ The cathode slurry was rolled onto an $\mathrm{Al}$ foil current collector, and assembled into a modified CR2032 coin cell with a Li anode, a $25 \mu \mathrm{m} \mathrm{PP/PE} / \mathrm{PP}$ separator (Celgard 2325), and using $1 \mathrm{M}$ of $\mathrm{LiPF}_{6}$ in $\mathrm{EC} / \mathrm{DMC}$ ( $1: 1$ by volume) as the electrolyte. A schematic view of this setup can be seen in Fig. 1.

The pristine LFS sample was characterized using SEM, XANES, and synchrotron radiation XRD. From SEM imaging and processing using Image $\mathrm{J}^{16}$ (Fig. 2a), the particle area was determined to be on average $1270 \pm 285 \mathrm{~nm}^{2}$. This reveals that the pristine material is composed of nanoparticles on the order of $\sim 40 \mathrm{~nm}$ and is in the form of aggregates. ${ }^{15}$ Synchrotron-radiation XRD

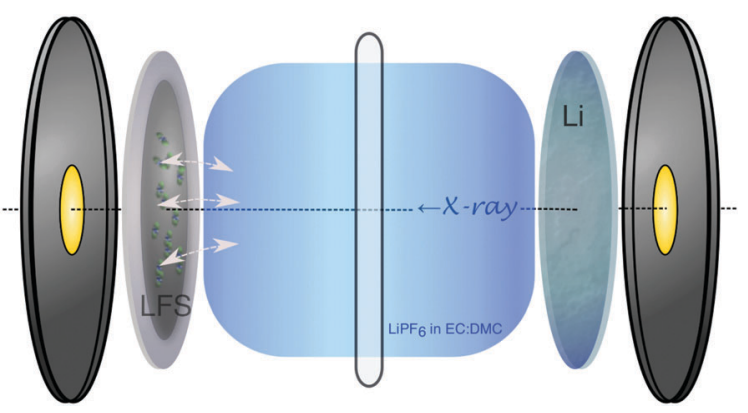

Fig. 1 Schematic of the in situ CR2032 coin cell with Kapton ${ }^{\circledR}$ windows used for XANES monitoring of the LFS-electrolyte interaction (depicted with arrows at the solid-electrolyte interphase). 
a)

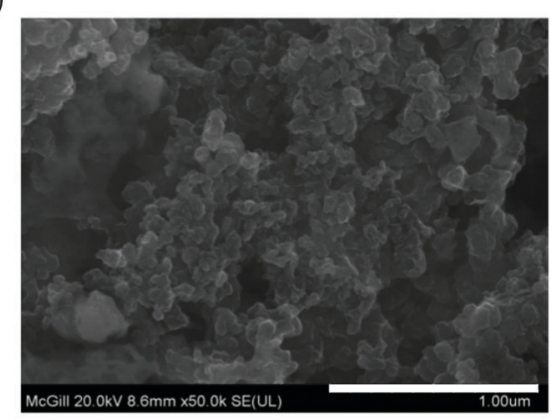

b)

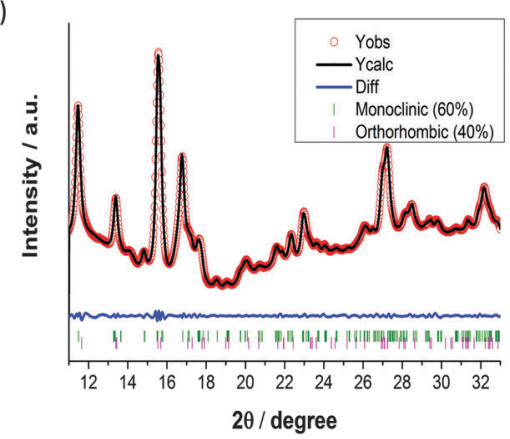

Fig. 2 Morphology and phase identification of the pristine LFS sample as characterized by SEM and SR-XRD; (a) SEM image taken at $20.0 \mathrm{kV}$ shows the aggregation of the LFS nanoparticles and (b) Rietveld refinement of the SR-XRD data, revealing an approximate phase mixture $3: 2$ of monoclinic and orthorhombic structures, respectively.

and XANES measurements were taken at the HXMA beamline at the Canadian Light Source (CLS). ${ }^{17}$ The CLS HXMA wiggler was running at $1.9 \mathrm{~T}$ and the CLS storage ring was with a $250 \mathrm{~mA}$ current. The $\mathrm{Si}(111)$ monochromator crystals in combination with an Rh coated pre-mono collimating mirror and a postmono toroidal focusing mirror were used. For the XANES measurements, both the incoming and transmitted X-ray intensity were monitored using ionization chambers filled with $\mathrm{He}$ gas, the fluorescence yield was monitored using a Lytle detector filled with $\mathrm{N}_{2}$ gas, and data were reduced using Athena; ${ }^{18}$ for XRD detection a mar345 image plate was used to record the Debye-Scherrer rings from a modified CR2032 cell (Fig. 1), and rings were integrated using fit2D. ${ }^{19}$ XANES was performed at the Fe K-edge and the XRD patterns were recorded at $17 \mathrm{keV}$ photon energy; energy calibration was performed by determining the first inflection point of standard Fe and Yt XANES, for the respective energies. The mar345 detector distance was calibrated using a NIST $\mathrm{LaB}_{6}$ powder mounted inside an in situ cell case, and fit2D's calibration function. The as-prepared material was found to be a mixture of approximately $3: 2$ of monoclinic $P 2_{1} / n$ $\left(\gamma_{\mathrm{s}}\right)$ and the "low temperature" orthorhombic Pmn $2_{1}\left(\beta_{\text {II }}\right)$ phases (Fig. 2b), respectively. ${ }^{20}$

In situ XANES measurements of the cathode were performed on the initial uncharged battery state (96 hours following the coin cell assembly during which LFS was in contact with $1 \mathrm{M}$ of $\mathrm{LiPF}_{6}$ in EC/DMC solvent) as well as after cycling the cell at the $\mathrm{C} / 20$ rate for a single $\mathrm{Li}$ extraction-insertion (formation) cycle. The "uncharged" cell is labelled $20 \mathrm{C}_{0}$ and the "cycled" cell, $20 \mathrm{D}_{\mathrm{f}}$.
These three samples correspond to the same electrochemical state, and represent the typical spectra we have measured for this material in these electrochemical states.

The normalized Fe K-edge XANES spectra in Fig. 3a show the results for the different LFS samples. The differences among the electronic structures of the three LFS samples are subtle but nevertheless noticeable. The first of which is that, the pristine sample (Fig. 3a and b black) shows well-resolved spectral features following the "white-line" area (i.e. the strongest peak area above the edge jump), compared to the other two samples: $20 \mathrm{C}_{0}$ and $20 \mathrm{D}_{\mathrm{f}}$, uncharged and cycled (formation cycle), respectively. This reflects the increase of structural disorder in samples $20 \mathrm{C}_{0}$ and $20 \mathrm{D}_{\mathrm{F} \cdot} \dagger$ For sample $20 \mathrm{C}_{0}$ this means that the contact of the LFS cathode with the electrolyte inside the coin cell (96 hours of storage time at room temperature) has triggered a spontaneous reaction resulting in a noticeable structural change in the cathode material. Upon cycling (formation cycle) however, the cathode $\left(20 D_{f}\right.$ sample) is seen to restore, in part, its local structure towards that of the pristine sample. This can be seen by comparing the post-white-line" region (Fig. 3b) and more clearly in the derivative spectra. The first derivatives of the XANES spectra for the three samples are shown in the insetof Fig. $3 \mathrm{~b}$, and were calculated using the central difference method. Here the restoration trend can be seen by following the peak at around $7125 \mathrm{eV}$. This corresponds to the inflection point just before the "white-line" region (where the spectra are free of any pre-edge effects caused by Jahn-Teller distortions). Specifically this derivative peak moves toward higher energy in the uncharged material and then returns to the same position as the pristine upon cycling.

The previous observations are echoed by the trend of the preedge features (Fig. 3c), that is the pristine sample has the lowest overall pre-edge intensity while the uncharged has the highest. This feature is reflective of the distortion of the $\mathrm{FeO}_{4}$ tetrahedron via the Jahn-Teller effect. ${ }^{21}$ Here the cycled cathode pre-edge intensity is between that of the other two, indicating again a certain level of structural restoration. In addition to the overall pre-edge intensity differences, Fig. $3 \mathrm{c}$ shows clearly the varying intensity distribution profiles among the three, which are related to the underlying spectral components of different oxidation species. $^{22}$ To facilitate further analysis, in Fig. 3c two lowtemperature reference phases have been included for the ferrous and ferric states of Fe. These are the tetrahedrally coordinated $\mathrm{Fe}^{2+} \mathrm{S}_{4}$ and $\mathrm{Fe}^{3+} \mathrm{S}_{4}$ found in rubredoxin. ${ }^{23}$ The ratio of the pre-edge features contributed by the ferrous and ferric states was fit using a linear combination ${ }^{18}$ of pre-edges from the $\mathrm{Fe}^{2+} \mathrm{S}_{4}$ and $\mathrm{Fe}^{3+} \mathrm{S}_{4}$ reference data. The fitting results are summarized in Fig. 4, each fit performed reported an $R$-factor ${ }^{18}$ less than 0.025 . The results show that the Fe site within the LFS matrix indeed became oxidized (likely with the reduction of the electrolyte) during the 96 hour interphasial interaction; upon cycling, this state was partially restored towards the original ferrous/ferric ratio in the pristine sample.

The relative spectral intensity increase of $\mathrm{Fe}^{3+}$ in the uncharged cathode compared to pristine (Fig. 4) is usually a direct fingerprint of the charge compensation process throughout a normal charging sequence. Here however, no external charging has been applied. 

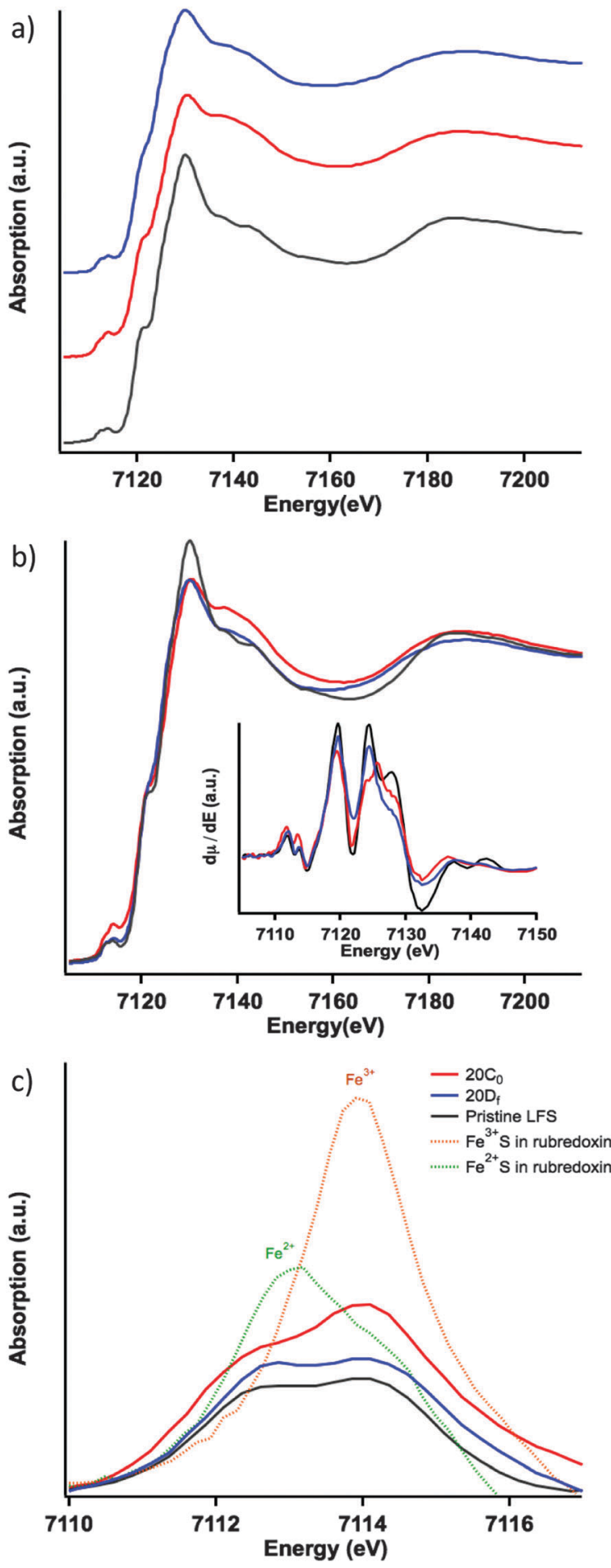

Fig. 3 XANES measurements of LFS in different charging states: (a) (black) pristine LFS, (red) $20 \mathrm{C}_{0}$ cathode inside the uncharged coin cell (LFS in contact with the $\mathrm{LiPF}_{6} \mathrm{EC} / \mathrm{DEC}$ electrolyte), and (blue) $20 \mathrm{D}_{\mathrm{f}}$ cathode after cycling at $1 / 20 \mathrm{C}$; (b) components from (a) overlaid for direct comparison; inset: derivative spectra highlighting the pre-edge - white-line region; (c) zoomed-in pre-edge region with background subtracted, also included are the reference spectra pre-edges of $\mathrm{FeS}_{4}$ in rubredoxin reduced and oxidized states, respectively.

In this case, in the absence of any applied current, this pre-edge profile variance indicates the delithiation of the material as a consequence of reduction of an electrolytic component.

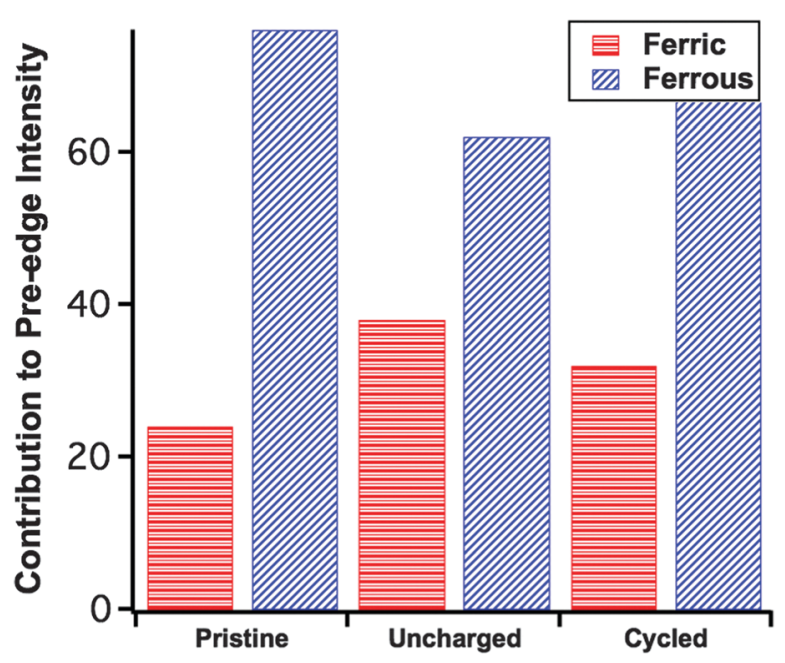

Fig. 4 Category plot of the $\mathrm{Fe}^{2+}$ and $\mathrm{Fe}^{3+}$ contribution to the pre-edge for the pristine material, uncharged $\left(20 C_{0}\right)$ and cycled $\left(20 D_{f}\right)$, as determined by a linear combination fit with $\mathrm{FeS}_{4}$ reference compounds: low-temperature XAS from molecular $\mathrm{FeS}_{4}$ in rubredoxin.

Previous research by Dippel et al. ${ }^{13}$ involving the ageing of the LFS material in a hot $\left(60{ }^{\circ} \mathrm{C}\right) \mathrm{LiPF}_{6}-\mathrm{EC} / \mathrm{DMC}$ organic electrolyte concluded that LFS converts under those conditions into $\mathrm{Li}_{2} \mathrm{SiF}_{6}$ with simultaneous dissolution of iron into the electrolyte. No delithiation or iron oxidation was reported as observed here. The same group in a follow up study ${ }^{11}$ commented that the electrolyte was thermally decomposed at $60{ }^{\circ} \mathrm{C}$ even in the absence of LFS hence the previous high temperature study appears to have observed reactions not necessarily operational in an actual coin cell that is typically used at lower temperatures. As per other electrode-electrolyte interphase systems ${ }^{14}$ it is likely that the reduction of the organic carbonate solvent molecules takes place on the surface of LFS leading to iron oxidation.

As a plausible mechanism for the observed spontaneous reaction between the $\mathrm{LiPF}_{6}-\mathrm{EC} / \mathrm{DMC}$ electrolyte and $\mathrm{Li}_{2} \mathrm{FeSiO}_{4}$ (LFS) we propose tentatively the following sequence. Fluoride anions (released from $\mathrm{LiPF}_{6}$ ) owing to their well-known propensity to form $\mathrm{Si}-\mathrm{F}$ groups (such as $\mathrm{SiF}^{6-}$ ) are envisaged to react with the surface of LFS as noted in Ensling et al.'s XPS analysis. ${ }^{12}$ Such Si-F interaction on the LFS surface would weaken the $\mathrm{Fe}^{\mathrm{II}}-\mathrm{SiO}_{4}$ bonding making iron(II) prone to oxidation via reduction of electrolyte components such as EC and DMC that is well established in the literature. ${ }^{13}$ In other words the oxidation of $\mathrm{Fe}(\mathrm{II})$ in this case is rendered feasible because of the F-induced weakening of the $\mathrm{Fe}^{\mathrm{II}}-\mathrm{Si}-\mathrm{O}_{x}$ bonding caused by the $\mathrm{Si}-\mathrm{F}$ interaction. Further detailed investigations would be needed to allow for a deeper understanding of this reaction and we are in the process of doing that.

Beyond the observation of the spontaneous reaction (while the cell is uncharged) another important revelation of this work is the finding that after the first charge-discharge cycle there is (at least in part) restoration of the LFS structure as evident by the oxidation state of iron. Thus the relative spectral intensity of $\mathrm{Fe}^{3+}$ was reduced from $\sim 37 \%$ at the "uncharged" state (i.e. following the spontaneous reaction) to approximately 
$32 \%$ at the discharged state (after the formation cycle) compared to $24 \%$ for the particular pristine sample used in this work (Fig. 4). It should be pointed out that the cycling process is known to produce a crystalline phase change in the pristine material, ${ }^{8,15}$ which could affect the overall intensity of the preedge features. The above analysis is concerned only with the relative ratio of $\mathrm{Fe}^{2+}$ and $\mathrm{Fe}^{3+}$ contributions to the overall preedge spectrum. What these results imply in terms of battery performance, however, is that though the reduction of the electrolyte does occur spontaneously and slightly increases the state-of-charge (SOC) of LFS during coin cell pre-cycling storage, the accompanying delithiation reaction is at least in part reversible. Given these findings further research is warranted not only in elucidating this complex interphasial reaction but also in assessing its role in the overall structural stability and loss of irreversibility during cycling of this important cathode material. The study of an alternative to F-containing electrolytes is equally important in this context.

In summary, in situ Fe K-edge XANES reveals a spontaneous interphasial reaction between the uncharged $\mathrm{Li}_{2} \mathrm{FeSO}_{4}$ cathode and the $\mathrm{LiPF}_{6}$-based electrolyte, manifested with the $\mathrm{Fe}^{2+} / \mathrm{Fe}^{3+}$ ratio variation from the pristine state. Subsequent cycling of the cathode material (formation cycle) is partially restorative in the Fe oxidation state.

The authors acknowledge assistance provided during data acquisition by Dr W. Chen and Dr C.-Y. Kim. Further acknowledgement and gratitude are extended to Dr L. Zhang (University of Nebraska-Lincoln) for providing the reference compound data. This work is supported through a Hydro-Québec/Natural Sciences \& Engineering Research Council of Canada (NSERC) Collaborative R\&D research grant. DTJ acknowledges Canadian NSERC support for the work done at the University of Guelph. Synchrotron radiation measurements were performed at the CLS, which is supported by CFI, NSERC of Canada, University of Saskatchewan, Government of Saskatchewan, Western Economic Diversification Canada, NRC Canada, and CIHR.

\section{Notes and references}

$\dagger$ Heterogeneity of unique local ionic environments offers on average a higher continuity of available states. In effect, XANES peak broadening can be due to the presence of multiple crystalline phases, or, as is the case here, from an increased local structure disorder within the same phase.

1 J. M. Tarascon and M. Armand, Nature, 2001, 414, 359-367.

2 J. B. Goodenough and K.-S. Park, J. Am. Chem. Soc., 2013, 135, 1167-1176.

3 M. E. Arroyo-de Dompablo, M. Armand, J. M. Tarascon and U. Amador, Electrochem. Commun., 2006, 8, 1292-1298.

4 A. Nytén, A. Abouimrane, M. Armand, T. Gustafsson and J. O. Thomas, Electrochem. Commun., 2005, 7, 156-160.

5 M. S. Islam, R. Dominko, C. Masquelier, C. Sirisopanaporn, A. R. Armstrong and P. G. Bruce, J. Mater. Chem., 2011, 21, 9811-9818.

6 D. Lv, J. Bai, P. Zhang, S. Wu, Y. Li, W. Wen, Z. Jiang, J. Mi, Z. Zhu and Y. Yang, Chem. Mater., 2013, 25, 2014-2020.

7 A. W. Brownrigg, G. Mountjoy, A. V. Chadwick, M. Alfredsson, W. Bras, J. Billaud, A. R. Armstrong, P. G. Bruce, R. Dominko and E. M. Kelder, J. Mater. Chem. A, 2015, 3, 7314-7322.

8 A. R. Armstrong, N. Kuganathan, M. S. Islam and P. G. Bruce, J. Am. Chem. Soc., 2011, 133, 13031-13035.

9 J. B. Goodenough and Y. Kim, Chem. Mater., 2010, 22, 587-603.

10 Y. Li, M. Bettge, B. Polzin, Y. Zhu, M. Balasubramanian and D. P. Abraham, J. Electrochem. Soc., 2013, 160, A3006-A3019.

11 C. Dippel, S. Krueger, V. Kraft, S. Nowak, M. Winter and J. Li, Electrochim. Acta, 2013, 105, 542-546.

12 D. Ensling, M. Stjerndahl, A. Nyten, T. Gustafsson and J. O. Thomas, J. Mater. Chem., 2009, 19, 82-88.

13 C. Dippel, S. Krueger, R. Kloepsch, P. Niehoff, B. Hoffmann, S. Nowak, S. Passerini, M. Winter and J. Li, Electrochim. Acta, 2012, 85, 66-71.

14 K. Xu, Chem. Rev., 2014, 114, 11503-11618.

15 X. Lu, H. Wei, H.-C. Chiu, R. Gauvin, P. Hovington, A. Guerfi, K. Zaghib and G. P. Demopoulos, Sci. Rep., 2015, 5, 8599.

16 C. A. Schneider, W. S. Rasband and K. W. Eliceiri, Nat. Methods, 2012, 9, 671-675.

17 D. T. Jiang, N. Chen and W. Sheng, AIP Conf. Proc., 2007, 879, 800-803.

18 B. Ravel and M. Newville, J. Synchrotron Radiat., 2005, 12, 537-541.

19 A. P. Hammersley, S. O. Svensson, M. Hanfland, A. N. Fitch and D. Hausermann, High Pressure Res., 1996, 14, 235-248.

20 C. Eames, A. Armstrong, P. Bruce and M. Islam, Chem. Mater., 2012, 24, 2155-2161.

21 G. Frank de, V. György and G. Pieter, J. Phys.: Condens. Matter, 2009, 21, 104207.

22 T. E. Westre, P. Kennepohl, J. G. DeWitt, B. Hedman, K. O. Hodgson and E. I. Solomon, J. Am. Chem. Soc., 1997, 119, 6297-6314.

23 L. Zhang, 2015, private communication. 